\title{
science angels - Nachwuchsförderung durch die Gemeinschaft des Verbands der Hochschullehrer für Betriebswirtschaft
}

\author{
Isabel Victoria Villeda, Henriette Houben, Ulf Brüggemann, Christian D. Schade und Tina Osteneck
}

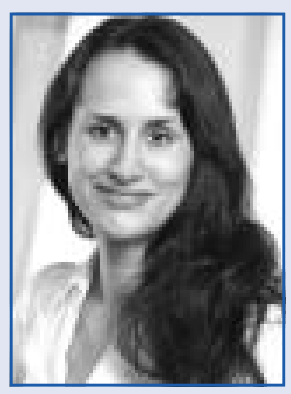

Dr. Isabel Victoria Villeda, Senior Consultant, Batten \& Company GmbH, Düsseldorf, sowie ehemaliges Mitglied der Nachwuchs AG im VHB. Bevorzugtes Forschungsgebiet: Marketing.

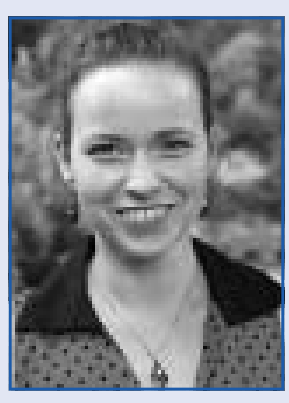

Prof. Dr. Henriette Houben, HumboldtUniversität zu Berlin und Mitglied der Nachwuchs AG im VHB. Bevorzugtes Forschungsgebiet: Empirische Steuerforschung.

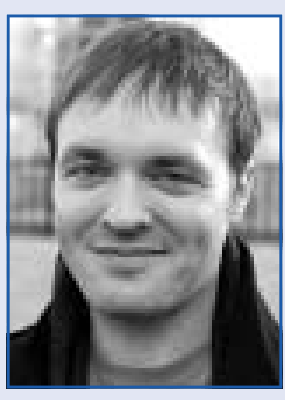

Prof. Dr. Ulf Brüggemann, Humboldt-Universität zu Berlin und Mitglied der Nachwuchs AG im VHB. Bevorzugtes Forschungsgebiet: Empirische Rechnungslegungsforschung.

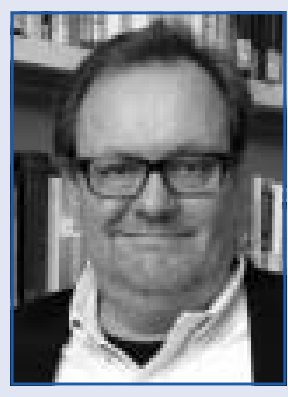

Prof. Dr. Christian D. Schade, HumboldtUniversität zu Berlin, Vorstandsmitglied des VHB, Ressort: Nachwuchs/ProDok und Leiter der Nachwuchs AG. Bevorzugte Forschungsgebiete: Behavioral Decision Making, Unterschiede im Entscheidungsverhalten von Männern und Frauen, Quantum Decision Making.

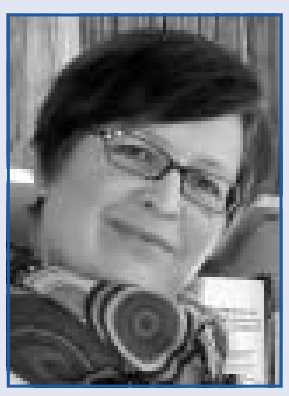

Dipl.-Kffr. Tina Osteneck, Geschäftsführerin des VHB.

Der Verband der Hochschullehrer für Betriebswirtschaft e.V. (VHB) hat ein neues Programm zur Förderung des wissenschaftlichen Nachwuchses entwickelt. Dieses trägt den Namen "science angels" und ist ein Coachingprogramm, in dem grundsätzlich auf die gesamte Expertise der deutsch-sprachigen BWL zurückgegriffen werden kann. Details werden im Beitrag erläutert.

Stichwörter: Coaching, Nachwuchsförderung, VHB, Science Angels

\section{Die science angels des VHB}

Nachwuchswissenschaftlerinnen und Nachwuchswissenschaftler in der BWL sind heutzutage mit großen Anforderungen und multiplen, teils unübersichtlichen Karrierewegen konfrontiert. Internationale Publikationen sind sehr wichtig, die Expertise dafür aber nicht einfach zu erlangen. Und die Voraussetzungen sind im Rahmen der unterschiedlichen Karrierewege einmal mehr, einmal weniger gegeben. Der BWL-spezifische Beratungsbedarf ist somit groß. Professionelle Coaches können in Ermangelung fachspezifischen Wissens nur sehr unzureichend Rat geben.

Seit 1921 gehört es zu den zentralen Anliegen des Verbands der Hochschullehrer für Betriebswirtschaft (VHB) e.V., die Betriebswirtschaftslehre als Universitätsdisziplin zu stärken, die Gemeinschaft der Mitglieder zu fördern sowie den wissenschaftlichen Nachwuchs zu unterstützen (http://vhbonline.org). Das Programm der science angels (www.science-angels.org), das der Vorstand zusammen mit der Arbeitsgruppe Nachwuchsförderung des VHB 
(http://vhbonline.org/einrichtungen/ag/ag-nachwuchs) Ende 2014 ins Leben rief, verbindet diese drei Kernziele des Verbands:

Als Coaching-Programm möchte es den wissenschaftlichen Nachwuchs stärken und ihm Experten aus der Gemeinschaft des $V H B$ an die Seite stellen. Diese Experten sollen in Form der science angels den Nachwuchs in der kritischen Karrierephase auf dem Weg zum Hochschullehrer unterstützen und ihm das Wissen der Gemeinschaft in persönlichen Gesprächen zur Verfügung stellen. Diese Gespräche bieten Nachwuchswissenschaftlerinnen und Nachwuchswissenschaftlern die Möglichkeit, über das Betreuungsumfeld an der eigenen Fakultät und innerhalb der eigenen Community hinaus Antworten zu konkreten wissenschaftlichen Fragestellungen zu bekommen, Publikationsstrategien zu diskutieren oder sich in Karrierefragen beraten zu lassen. Das ist heutzutage besonders wichtig, da das erfolgreiche Publizieren immer bedeutsamer und der Weg zum Hochschullehrer durch die Vielzahl möglicher Karrierepfade immer unübersichtlicher geworden sind.

Neben diesen wertvollen Impulsen für Nachwuchswissenschaftlerinnen und Nachwuchswissenschaftler bietet das Programm etablierten Experten die Möglichkeit, ihr Wissen und ihre Erfahrungen gezielt weiterzugeben und dafür zu sorgen, dass die Qualität der Forschung und Lehre in Deutschland gefördert und die Betriebswirtschaftslehre als wissenschaftliche Disziplin gestärkt werden. Nicht zuletzt soll durch die intensive Zusammenarbeit der verschiedenen wissenschaftlichen Generationen die Vernetzung und das Gemeinschaftsgefühl der Verbandsmitglieder weiter gestärkt werden.

Für die Entstehung und den weiteren Ablauf des Coaching-Verhältnisses stellt der VHB seine Infrastruktur sowie einen gut definierten organisatorischen und strukturierten Rahmen zur Verfügung. Der $V H B$ agiert als Vermittler zwischen Nachwuchswissenschaftlerinnen und Nachwuchswissenschaftlern und einem, aus deren Sicht bevorzugten, Experten in Bezug auf eine bestimmte Fragestellung. Dabei verpflichtet sich der $V H B$ zu den Grundprinzipien von Vertraulichkeit und Freiwilligkeit: Die vertrauliche Anfrage der Nachwuchswissenschaftlerin oder des Nachwuchswissenschaftlers wird von der $V H B-G e-$ schäftsstelle zunächst anonym an die angefragten $V H B$ Expertinnen und -Experten weitergeleitet. Erst nachdem sich diese grundsätzlich bereit erklärt haben, als science angel ein vertrauliches Gespräch zu führen, werden Namen und nach Möglichkeit die zu behandelnde Fragestellung weitergegeben. Um ein optimales Coaching-Klima zu gewährleisten, wird dem $V H B$-Experten zu diesem Zeitpunkt erneut die Möglichkeit eingeräumt, das Gespräch abzulehnen und sich so ganz bewusst für die konkreten Anforderungen des jeweiligen Gesprächs zu entscheiden. Sowohl in der Vermittlung als auch in der Folge eines Coaching-Verhältnisses unterliegt der Austausch zwischen den science angels und dem wissenschaftlichem Nachwuchs absoluter Diskretion.

\section{Abgrenzung zu existierenden Coaching- Programmen}

Coaching-Programme gewinnen im Wissenschaftsbetrieb zunehmend an Bedeutung. Unsere Recherchen haben ergeben, dass viele Universitäten im deutschsprachigen Raum bereits eigene Programme anbieten. Diese Programme können in der Regel allerdings nur von Hochschulangehörigen in Anspruch genommen werden und sind daher, was das inhaltliche Spektrum anbelangt, naturgemäß begrenzt, oder sie sind Teil der Frauenförderungsinitiative und stehen dann dem männlichen Nachwuchs nicht zur Verfügung. Außerdem wird gemäß unserer Recherchen seitens der Universitäten ein hoher Bedarf an begleitender Unterstützung des wissenschaftlichen Nachwuchses (und das gilt auch und gerade in der BWL) gesehen. Im Gegensatz dazu ist das science angels-Programm hochschulübergreifend und geschlechterunabhängig organisiert. Dies ermöglicht den gecoachten Nachwuchswissenschaftlern, über ihre science angels wertvolle Einsichten in die Gepflogenheiten anderer Universitäten $\mathrm{zu}$ gewinnen, aber auch mögliche Besonderheiten und Herausforderungen der eigenen Hochschule mit einem neutralen Außenstehenden offen $\mathrm{zu}$ besprechen. Ein weiteres besonderes Merkmal des science angels-Programms ist, dass potenzielle Coaches den Nachwuchswissenschaftlern nicht zugeordnet, sondern von diesen ausgewählt werden können. Dies schafft beste Voraussetzungen für ein erfolgreiches Coaching durch die science angels, das den Erwartungen der Nachwuchswissenschaftler entspricht.

Um dies noch einmal zu betonen: Durch die direkte Verbindung zum $V H B$ sind nahezu alle universitären Hochschullehrer aus dem gesamten Spektrum der BWL potenzielle science angels. Dem Nachwuchs in der BWL geht es nicht in erster Linie um Fragen, die professionelle Coaches beantworten können (auch diese Fragen sind natürlich wichtig, und auch ein professionelles Coaching kann für eine Nachwuchswissenschaftlerin und einen Nachwuchswissenschaftler wichtig sein!), ihnen geht es um inhaltliche Fragen, zu Methoden, zu Theorien, zu Publikationsstrategien, zu der speziellen Ausrichtung und den Anforderungen eines bestimmten Journals, etc. Diese Fragen können nur von Experten beantworten werden, die selbst Teil des Systems und seiner Anforderungen sind.

\section{Ziele des science angels-Programms}

Das Konzept der science angels verfolgt vorrangig sechs Ziele, die in die Säulen der Förderung des wissenschaftlichen Nachwuchses, der Stärkung der Gemeinschaft der Mitglieder und der Stärkung der Betriebswirtschaftslehre als Universitätsdisziplin eingeteilt werden können (vgl. $A b b .1)$. Die Förderung des wissenschaftlichen Nachwuchses ist dabei das zentrale Ziel. Die beiden weiteren Säulen unterstützen dieses Ziel, da der Nachwuchs Teil der Gemeinschaft ist und für eine nachhaltige Perspektive der BWL als Universitätsdisziplin steht. 


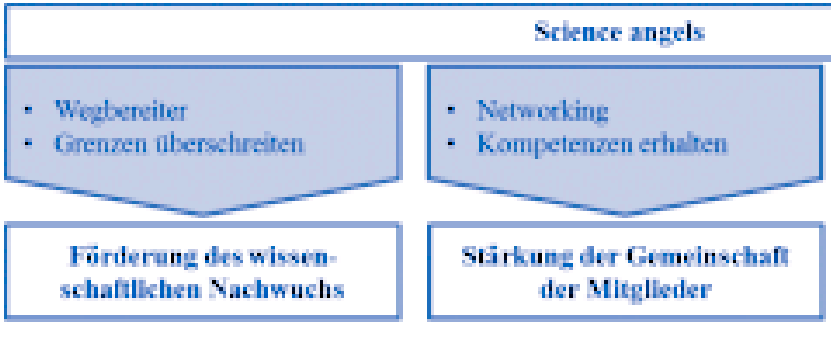

\subsection{Förderung des wissenschaftlichen Nachwuchses}

\subsubsection{Wegbereiter}

Das Coaching durch science angels ist für viele Phasen des Nachwuchsweges wichtig. Dies sei hier (ohne Anspruch auf Vollständigkeit) einmal idealtypisch beschrieben. Zunächst ergibt sich Coachingbedarf für die frühe Phase: Hier hat sich der wissenschaftliche Nachwuchs gerade bereit erklärt, den durch Publikationsdruck wettbewerbsintensiven und oft unübersichtlichen Weg einer wissenschaftlichen Karriere einzuschlagen. Hinzu kommt oft noch die Unsicherheit über den eigenen Karriereweg durch das Fehlen einer Tenure-Track-Option (einige Universitäten ändern dies zurzeit, aber dies betrifft im Moment nur wenige Nachwuchswissenschaftlerinnen und Nachwuchswissenschaftler). Erneut ergibt sich Coachingbedarf in der heißen Phase der inhaltlichen Positionierung, oft im Konflikt zwischen eigenen Interessen und den (scheinbar unausweichlichen) Anforderungen des Marktes. Dann ergibt sich ein Coachingbedarf noch einmal im eigentlichen Publikationsprozess und vielleicht später noch einmal, wenn es um Strategien im Zuge von Bewerbungen auf Stellen geht. In all diesen Phasen will der $V H B$ dem Nachwuchs beistehen und ihm in Form der science angels echte Experten zu Karrierewegen, zu Publikationsstrategien, zur eigenen Positionierung als Forschender und Lehrender, zur Auswahl des eigenen Karrierepfads und zu konkreten wissenschaftlichen Fragen zur Seite stellen.

Mit anderen Worten: Ein Coaching kann dazu dienen, sich über eine zu den persönlichen Präferenzen und Kompetenzen passende Positionierung in der Wissenschaftslandschaft klar zu werden und individuelle Perspektiven im Wissenschaftsbetrieb aufzuzeigen. Zu Beginn einer Karriere in der Forschung und Lehre mögen ein Erfahrungsaustausch oder ganz konkrete Best Practice Überlegungen den eigenen Veröffentlichungsweg weisen, später können Gespräche mit erfahrenen $V H B$-Mitgliedern wichtige Impulse bei oft sehr spezialisierten wissenschaftlichen Fragestellungen geben und so dem Nachwuchswissenschaftler den Weg in eine exzellente betriebswirtschaftliche Forschung und Lehre und damit zum Hochschullehrer soweit möglich erleichtern. Das Know-how der BWL-Hochschullehrer in Bezug auf Erfolgsstrategien bei Bewerbungen und Berufungsvorträgen kann dann ebenfalls eine wichtige Rolle spielen.

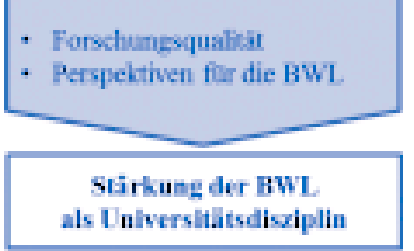

Abb. 1: Ziele des science angelsProgramms

\subsubsection{Grenzen überschreiten}

Nachwuchswissenschaftlerinnen und Nachwuchswissenschaftler benennen selber, von welchem Experten oder welcher Expertin sie oder er sich ein Coaching erwünschen. Diese Experten können frei aus den Reihen der erfahrenen $V H B$-Mitglieder gewählt werden.

Hierdurch soll zum Ersten eine optimale Passung zwischen dem Anliegen des Nachwuchswissenschaftlers und dem science angel garantiert werden. Zum Zweiten soll der Nachwuchswissenschaftler die Möglichkeit haben, auBerhalb seines eigenen bereits existierenden wissenschaftlichen Umfelds Expertise zu akquirieren. So werden die Grenzen zwischen den einzelnen Forschungsgruppen aufgeweicht und Erfahrungen über das enge Hochschulumfeld hinaus ermöglicht. Grenzen können aber auch dadurch überschritten werden, dass die Coaches selbst von neuen Herangehensweisen oder der Begeisterung des wissenschaftlichen Nachwuchses motiviert werden und auch auf diesem Wege von den Gesprächen profitieren.

\subsection{Stärkung der Gemeinschaft der Mitglieder}

\subsubsection{Networking}

Das science angels-Programm dient als Anstoß, die Vernetzungen zwischen den Wissenschaftlerinnen und Wissenschaftlern im Verband weiter zu stärken. Durch das Programm werden oft noch empfundene Hemmnisse bei der Ansprache zwischen den Wissenschaftsgenerationen überwunden, vermeintliche Treuepflichten aufgeweicht und somit aus einer neuen Freiheit und Kreativität der Forschung ganz neue Impulse eingebracht. Auch wenn das Coaching zeitlich begrenzt ist, kann es als Anstoß für längerfristige Vernetzungen dienen und so die Vernetzung innerhalb des $V H B$ insgesamt fördern.

\subsubsection{Kompetenzen erhalten}

Das science-angels-Programm will die Gemeinschaft nicht nur in Form einer intensiven Vernetzung der Mitglieder stärken. Es etabliert auch ein offizielles Instrument, um das Wissen und die Erfahrungen der Gemeinschaft gezielt auch an den Nachwuchs außerhalb des eigenen, engen Netzwerks weiterzugeben und so die Kompetenzen der Gemeinschaft dauerhaft zu erhalten. 


\subsection{Stärkung der Betriebswirtschaftslehre als Universitätsdisziplin}

\subsubsection{Forschungsqualität}

Durch vertrauensvolle persönliche Gespräche zwischen Wissenschaftlern insbesondere zu Forschungsfragen und Publikationsstrategien kann das Programm die Forschungseffizienz in der Betriebswirtschaftslehre erhöhen. Dabei können die Anstöße innerhalb einer Disziplin oder aber zwischen Disziplinen erfolgen und so das Spektrum der Kompetenzen voll ausschöpfen.

\subsubsection{Perspektiven für die Betriebswirtschaftslehre}

Mag es auch pathetisch klingen, so ist der heutige wissenschaftliche Nachwuchs die Zukunft der Forschung, Lehre und der Betriebswirtschaftslehre als Wissenschaftsdisziplin per se. Die Betriebswirtschaftslehre wird als wissenschaftliche Disziplin durch die Sicherung der Forschungs- und Lehrqualität des eigenen Nachwuchses nachhaltig gestärkt. Die effiziente Nutzung des Wissenspotenzials der deutschsprachigen BWL in der Nachwuchsausbildung ist dabei hilfreich, die Wettbewerbsfähigkeit der deutschsprachigen BWL gegenüber anderen Wissenschaftsdisziplinen und gegenüber konkurrierenden Angeboten im Ausland zu stärken. Das Programm bietet somit einen wichtigen Beitrag für den Forschungs- und Wissenschaftsstandort insgesamt sowie für den Erhalt und die Weiterentwicklung eines funktionierenden Wirtschaftssystems im deutschsprachigen Raum.

\section{Ablauf und Rolle des VHB}

Das science angels-Programm ist organisatorisch bei der Geschäftsstelle des $V H B$ angesiedelt. Alle Informationen sowie Formulare zum Programm finden sich auf der Seite des VHB (http://vhbonline.org/nachwuchs/science-angels) sowie inhaltsgleich auf der Seite der science angels (www.science-angels.org).

\subsection{Anfrage eines Coachings bei der Geschäftsstelle des $V H B$}

Der erste Schritt zu einem Coaching erfolgt durch die Person der Nachwuchswissenschaftlerin bzw. des Nachwuchswissenschaftlers (vgl. $A b b$. 2). In der Anfrage für ein Coaching bei der Geschäftsstelle des $V H B$ werden bis zu drei Coaches genannt, wobei eine Präferenzordnung genannt werden soll. Das gewünschte Coaching-Thema kann bei dieser ersten Anfrage - muss aber nicht - angegeben werden.

\subsection{Kontaktaufnahme der Geschäftsstelle des VHB mit dem/den gewünschten Coach(es)}

Die VHB-Geschäftsstelle kontaktiert den auf der Präferenzliste an erster Stelle stehenden Coach mit der Frage, ob er oder sie grundsätzlich bereit ist, zum gegenwärtigen Zeitpunkt ein Coaching zu übernehmen und mit der Bitte, die namentliche Anfrage vertraulich zu behandeln. Bei einer positiven Antwort des Coaches werden ihm Namen und Coaching-Thema genannt. Sofern der Coach sich in der Lage sieht, dieser Person zum gewünschten Thema als Coach zur Verfügung zu stehen, erklärt er sich zu einem Coaching-Gespräch bereit. Die Nachwuchswissenschaftlerin bzw. der Nachwuchswissenschaftler wird erst im Anschluss daran über die Entscheidung informiert.

Wird von dem erstgewünschten Coach ein Coaching allgemein oder im konkreten Fall abgelehnt, kontaktiert die Geschäftsstelle des $V H B$ analog zum vorherigen Vorgehen den zweit- und ggf. drittgewünschten Coach. Sollte keiner der gewünschten Coaches zur Verfügung stehen, wird die Nachwuchswissenschaftlerin bzw. der Nachwuchswissenschaftler informiert. Es besteht dann die Möglichkeit, erneut ein Coaching beim VHB zu beantragen. Dabei können zu einem späteren Zeitpunkt dieselben Wunsch-Coaches erneut oder aber andere Wunsch-Coaches genannt werden.

In allen Fällen verpflichtet sich der angefragte Coach zu Stillschweigen über die Person der Nachwuchswissenschaftlerin bzw. des Nachwuchswissenschaftlers und das betreffende Anliegen sowie zur Einhaltung der Vertraulichkeitsregelungen.

\subsection{Coaching-Gespräch}

Die konkrete Terminvereinbarung findet zwischen dem Coach und dem Nachwuchswissenschaftler statt. Die vom $V H B$ angesetzte Regeldauer für das persönliche Gespräch beträgt 90 Minuten. Im gegenseitigen Einverständnis kann dabei auch eine längere Gesprächszeit vereinbart werden. Das Gespräch findet einmalig statt, im Idealfall von Angesicht zu Angesicht. Natürlich können sich aus pragmatischen Gründen auch andere Varianten, etwa ein Skype-Gespräch oder ein Telefonat, anbieten. Das Gespräch unterliegt selbstverständlich ebenfalls den strengen Vertraulichkeitsregeln des science angels-Programms. Diese Regeln besagen u. a., dass alles zwischen den Personen Besprochene ohne die explizite Zustimmung beider Parteien den Raum nicht verlässt, dass ein Coaching-Gespräch keine weiteren Ansprüche wie z. B. eine Fortsetzung der Beziehung oder Ko-Autorenschaft begründet und dass ein wertschätzender, respektvoller Ton unter den Anwesenden gepflegt wird, der auch die Aussagen zu ggf. thematisierten Dritten mit einschließt.

\subsection{Zertifikate für Coaches}

Nach Beendigung des Coaching-Gesprächs erhält der Coach ein science angel-Zertifikat. Hiermit wird die besondere Verantwortung, die der Coach für den Nachwuchs übernimmt, seitens des $V H B$ bestätigt und dokumentiert. Der $V H B$ setzt dieses Zertifikat bewusst als Instrument ein, um die Bedeutung der Übernahme von Verantwortung für den wissenschaftlichen Nachwuchs nach außen zu tragen. Er verfolgt damit auch das Ziel, zu signalisieren, dass ein solches Engagement einen wichtigen Teil des umfangreichen Leistungskatalogs eines Hochschullehrers - über das reine (Eigen-) Publikationserfordernis hinaus - darstellt. 


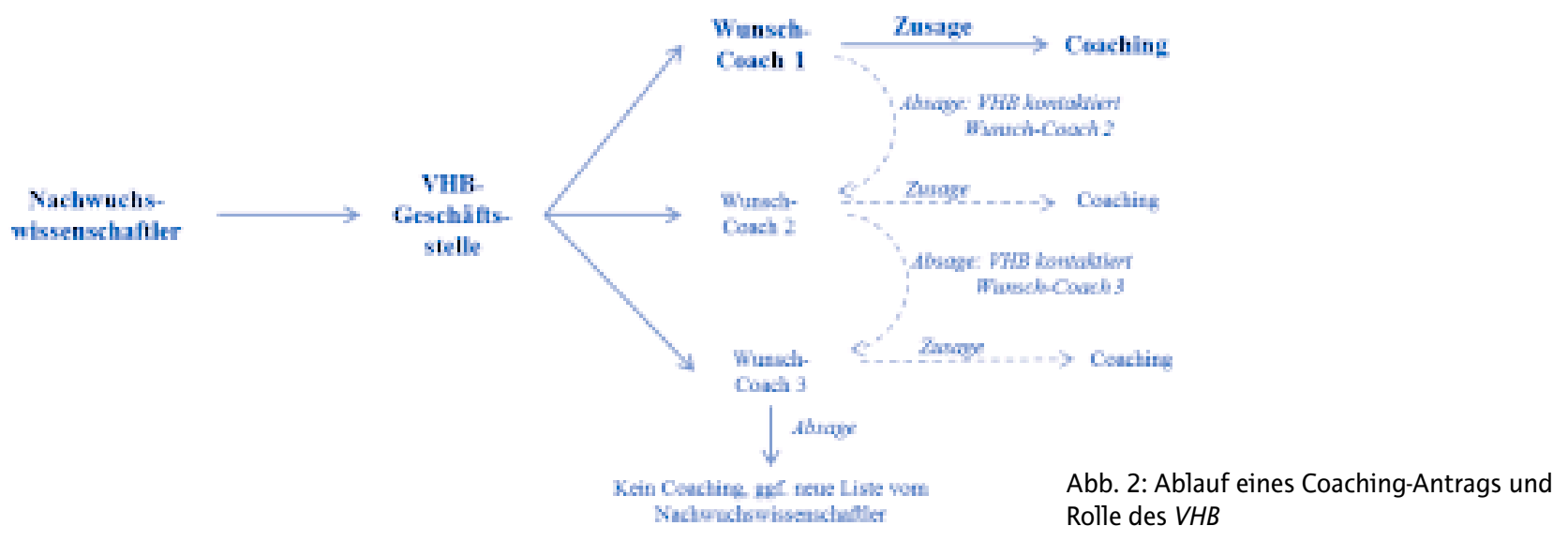

Das Zertifikat enthält weder den Namen der gecoachten Person noch ihr Anliegen.

\section{Begründer des Konzepts: VHB-Arbeitsgruppe Nachwuchsförderung}

Das science angels-Programm wurde von der $\mathrm{VHB}$-Arbeitsgruppe Nachwuchsförderung in der Zusammensetzung der Jahre 2013 und 2014 entwickelt: Prof. Dr. Christian D. Schade (Professur für Entrepreneurial and Behavioral Decision Making an der Humboldt-Universität $z u$ Berlin), Prof. Dr. Henriette Houben (Inhaberin der Ernst \& Young Stiftungs-Juniorprofessur für Quantitative Betriebswirtschaftliche Steuerlehre an der Humboldt-Universität zu Berlin) und Dr. Isabel Victoria Villeda (Senior Consultant, Batten \& Company GmbH, Düsseldorf). Im Jahre 2015 fand ein Wechsel von Dr. Victoria Villeda zu Prof. Dr. Ulf Brüggemann (damals Postdoktoraler Forscher und jetzt Professor am Institut für Rechnungswesen und Wirtschaftsprüfung an der Humboldt-Universität zu Berlin) statt, der ebenfalls zur Erstellung dieses Artikels beigetragen hat. Die Geschäftsstelle des $V H B$ hat wesentlich bei der konkreten Ausgestaltung des Programms mitgewirkt.
Die Arbeitsgruppe entwickelt gemeinsam Konzepte zur Förderung des wissenschaftlichen Nachwuchses wie z. B. das science angels-Programm. Weitere Aufgaben liegen in der Mitorganisation der jährlichen Pfingsttagung des $V H B$, konkret der Programmpunkte Brainpool, Karrierepanel und Nachwuchsstammtisch. Im Rahmen des Brainpools werden Themen erörtert, die den Nachwuchs aktuell beschäftigen. Bei der Pfingsttagung 2014 wurde beispielsweise über die Vor- und Nachteile von „Out of the Box“Forschung gesprochen. Im Karrierepanel werden karrierespezifische Fragen aus den Reihen der Nachwuchswissenschaftler mit einem Podium von entsprechenden Experten diskutiert. Beim Nachwuchsstammtisch kann der Nachwuchs in entspannter Atmosphäre seine Vernetzung vorantreiben und Kontakte zu Forschern anderer Teildisziplinen und anderer Universitäten knüpfen.

\section{Literatur}

Klinkhammer, M., A. Frohnen, Zwischen Exzellenz und Existenzsicherung - Aktuelle Herausforderungen im Coaching von Wissenschaftlerinnen und Wissenschaftlern, in: R. Wegener, A Fritze, M. Leobbert (Hrsg.), Coaching-Praxisfelder, Forschung und Praxis im Dialog: Publikation zum zweiten internationalen Coaching-Fachkongress, Berlin 2015, S. 178-190. 